\title{
FIP/AAPS Joint Workshop Report: Dissolution/In Vitro Release Testing of Novel/Special Dosage Forms
}

\author{
Cynthia K. Brown, ${ }^{1,11}$ Horst Dieter Friedel, ${ }^{2}$ Amy R. Barker, ${ }^{1}$ Lucinda F. Buhse, ${ }^{3}$ Susanne Keitel, ${ }^{4}$ Todd L. Cecil, ${ }^{5}$ \\ Johannes Kraemer, ${ }^{6}$ J. Michael Morris, ${ }^{7}$ Christos Reppas, ${ }^{8}$ Mary P. Stickelmeyer, ${ }^{1}$ \\ Chikako Yomota, ${ }^{9}$ and Vinod P. Shah ${ }^{10}$
}

Received 28 March 2011; accepted 27 April 2011; published online 18 June 2011

KEY WORDS: In vitro release testing; dissolution; novel dosage forms; special dosage forms

In 2003, the FIP Dissolution Working group published a position paper on dissolution/drug release testing for special/novel dosage forms that represented the scientific opinions of many experts in the field at that time (1). The position paper has supported activities, programs, and decisions in the scientific, technical, and regulatory community. Due to the rapid evolution of new practices and techniques for in vitro testing, the FIP Special Interest Group (SIG) on Dissolution/Drug Release decided to revise the previous paper and added proposals for further harmonization of in vitro release testing practices for different pharmaceutical dosage forms. This article represents the current updates to the previously published paper. This revision has been aligned to coincide with the USP taxonomy including route of administration, intended site of drug release, and dosage form. The revised paper includes information from current literature, expert discussions, and presentations from recent workshops $(2,3)$. The authors

Please send comments to the Co-Chairs of the FIP Dissolution/Drug Release Special Interest Group:

Cynthia K. Brown and Horst-Dieter Friedel

The findings and conclusions in this article have not been formally disseminated by the Food and Drug Administration or other licensing authorities and should not be construed to represent any agency determination or policy.

${ }^{1}$ Eli Lilly and Company, Indianapolis, Indiana, USA.

${ }^{2}$ Bayer HealthCare AG, Berlin, Germany.

${ }^{3}$ Food and Drug Administration/CDER/OPS, St. Louis, Missouri, USA.

${ }^{4}$ EDQM, Council of Europe, Strasbourg, France.

${ }^{5}$ United States Pharmacopeia, Rockville, Maryland, USA.

${ }^{6}$ PHAST, Homburg, Germany.

${ }^{7}$ Irish Medicines Board, Dublin, Ireland.

${ }^{8}$ National \& Kapodistrian University of Athens, Panepistimiopolis, Greece.

${ }^{9}$ National Institute of Health Science, Tokyo, Japan.

${ }^{10}$ FIP Scientific Secretary, The Hague, Netherlands.

${ }^{11}$ To whom correspondence should be addressed. (e-mail: brownck@lilly.com) acknowledge and expect further updates to be made as additional progress is made in the relevant areas. Thus, comments and additional contributions are welcome and may be considered for the next revision of the position paper.

\section{CONCEPT OF DISSOLUTION/DRUG RELEASE TESTING}

In the pharmaceutical industry, dissolution testing is an important tool in both drug development and quality control. Although initially developed for immediate release (IR) solid oral dosage forms and then for modified release solid oral dosage forms, the application of dissolution testing has expanded to a variety of "novel" or "special" dosage forms. As these formulations have become more prevalent due to complexities of drug delivery, there has been an increased development of modified testing methods to characterize the in vitro release of these dosage forms.

For orally administered IR solid drug products, it is customary to refer to the test as a "dissolution" test, since the intention is that the drug dissolves rapidly in the test medium. For non-oral dosage forms such as topical and transdermal delivery systems, suppositories, and others, the test is referred to preferably as a "drug release" or "in vitro release" test procedure. As novel/special dosage forms exhibit significant differences in formulation design, which in turn leads to very different physicochemical and release characteristics, it is not possible to devise a single test system which could be used to study the drug release properties, techniques, and purpose of each special dosage form. Rather, different apparatus, procedures, and techniques are employed on a case by case basis, and the method may be specific to the dosage form category, formulation type, or even to a particular individual product.

The general principles of dissolution tests for solid oral dosage forms should also be applicable to many in vitro dissolution/drug release tests for novel/special dosage forms. The ultimate goal of these tests is analogous to that for solid 
oral dosage forms, i.e., to use the test for the biopharmaceutical characterization of the drug product, and as a discriminating tool to assure consistent product quality within a defined set of specification criteria.

Different types of dosage forms and appropriate apparatus and testing methods used for drug release testing are discussed below. For several novel/special dosage forms, the methodology is well evolved, and specific recommendations can be made for drug release testing, e.g., for suspensions, orally disintegrating tablets, chewable tablets, suppositories, transdermal patches, and semisolid topical dosage forms (creams, ointments, and gels). However, as for conventional oral dosage forms, there may be specific formulations in the abovementioned categories for which the evolved methods are not applicable. In several other instances, e.g., chewing gums, powders, granules, inhalations, solid dispersions, microparticulate formulations, and implants, more methods development, and refinements will be required before a final recommendation for a standardized drug release method can be made. For these dosage forms, a brief summary of the state-of-the-art knowledge is provided to guide further development. Due to the different characteristics of the novel/special dosage forms and their sites and modes of administration, it is essential that apparatus selection, composition of the dissolution medium, agitation (flow rate), and temperature be given appropriate consideration during method design. In instances where a compendial (e.g., European Pharmacopoeia (Ph. Eur.), Japanese Pharmacopoeia (J.P.), United States Pharmacopeia (USP)) method is not employed for the in vitro drug release testing, the experimental test conditions, qualifications, and validation steps should conform to those discussed in the International Federation of Pharmaceutical Sciences (FIP) and US Food and Drug Administration (FDA) and other authorities guidance on dissolution testing (4,5).

In general, compendial apparatus and methods should be used as a first approach in drug development. To avoid unnecessary proliferation of equipment and method design, modifications of compendial equipment and development or use of alternative equipment should be considered only when it has been proven that compendial setup does not provide meaningful data for a given (new) dosage form. Qualification and validation efforts would include those quoted above $(4,5)$ and would be expected to demonstrate that the new method is scientifically sound and guarantees accurate, precise, and reproducible data. Appropriate method development and validation assures acceptable drug product quality and allows for some interpretation of the product's in vivo performance.

In some cases, the method used in the early phase of product/formulation development could be different from the final test procedure utilized for the control of the product quality. Indeed, methods used for formulation screening or understanding of the release mechanism may simply be impractical for a quality control environment. It is essential that with the accumulation of experience, the early method be critically reevaluated and potentially simplified, giving preference to compendial apparatus. While the methods may be related in the information they offer, the final method may not necessarily imitate the in vivo environment. The final quality control method should test the key performance indicators of the formulation.

\section{DOSAGE FORM TAXONOMY}

Medicinal drug products are administered in the body by one of the five routes of drug administration: oral, topical/dermal, mucosal, parenteral, and inhalations (6). For each route of the drug administration, two types of tests are proposed: (1) the product quality tests and (2) the product performance test. The product quality tests include identity, strength, uniformity of dosage units, purity, etc., whereas a product performance test in most cases constitutes a drug release test, analogous to a dissolution test. Most of the tests discussed in this paper are product performance tests. The five routes of drug administration with their intended sites of drug release and examples are included in Table I.

Grouping products based on taxonomy, as well as quality and performance tests helps identify where the standards of practice in the industry and expectations of regulators have begun to coincide. It is also possible to more easily identify areas that are in need of development and further discussion. In the remainder of this document, an evaluation of the state of the industry is examined for special dosage forms within this classification system.

\section{DOSAGE FORMS FOR WHICH A SPECIFIC METHOD CAN BE RECOMMENDED}

\section{Solid Oral Dosage Forms}

\section{Orally Disintegrating or Orodispersible Tablets}

Orally disintegrating tablets (ODT) are designed to rapidly disintegrate in the oral cavity. The December 2008 FDA Guidance for Industry (7) recommends a disintegration time of no more than $30 \mathrm{~s}$ and a tablet weight of less than $500 \mathrm{mg}$. The Ph. Eur. (7.3) calls these products orodispersible tablets and defines them as having a disintegration time within $3 \mathrm{~min}$. The administration of ODTs may not inherently result in a faster therapeutic onset, but can circumvent problems such as difficulty in swallowing traditional solid oral dosage forms like tablets and capsules, and can improve ease of use of a product by providing a means of drug delivery without water or liquids. ODTs can have buccal and/ or GI absorption, so both dissolution testing and disintegration testing are important.

In vitro dissolution testing should follow the principles of solid oral dosage forms (tablets) or suspensions (1). The rotating paddle would be the method of first choice. Many formulations float or form pulpy masses so a potential difficulty for in vitro dissolution testing may arise from floating particles. A single point specification is considered appropriate for ODTs. A disintegration test may be used in lieu of a dissolution test if it is shown to be discriminating (ICH Q6A Decision tree 7).

ODTs often make use of taste masking technologies such as coating of drug particles to improve palatability. A low dissolution rate in the first few minutes may be an indicator of coating for taste masking purposes and may not have any relevance in terms of the product's biopharmaceutical properties. Taste masking properties can be evaluated by dissolution and thus avoid organoleptic testing. 
Table I. Taxonomy Summary

\begin{tabular}{|c|c|c|}
\hline Route of administration & Intended site of release & Dosage form examples \\
\hline Oral & Gastro intestinal tract & $\begin{array}{l}\text { Solid dosage forms } \\
\text { Tablets } \\
\text { Capsules } \\
\text { Disintegrating tablets } \\
\text { Oral/dispersible } \\
\text { Chewable (tablets and gums) } \\
\text { Liquid filled capsules } \\
\text { Powders } \\
\text { Granules } \\
\text { Solid solutions } \\
\text { Solid dispersions } \\
\text { Liquid dosage forms }\end{array}$ \\
\hline Topical/dermal & Skin & $\begin{array}{l}\text { Transdermal delivery system (patch) } \\
\text { Semi solid dosage forms } \\
\text { Gels } \\
\text { Creams, lotions and ointments }\end{array}$ \\
\hline \multirow[t]{7}{*}{ Mucosal (Local or systemic) } & Oral & $\begin{array}{l}\text { Chewing gum } \\
\text { Thin dissolvable films (wafers) }\end{array}$ \\
\hline & Ophthalmic & $\begin{array}{l}\text { Implants } \\
\text { Liquids } \\
\text { Suspensions }\end{array}$ \\
\hline & Rectal & Suppositories \\
\hline & Intrauterine & Device \\
\hline & Otic & Liquids \\
\hline & Vaginal & $\begin{array}{l}\text { Suppositories } \\
\text { Semisolids } \\
\text { Thin dissolvable films (wafers) }\end{array}$ \\
\hline & Urethral & \\
\hline Parenterals & Bodily tissues and fluids & $\begin{array}{l}\text { Microparticulate systems } \\
\text { Subcutaneous liposomes } \\
\text { Intramuscular drug-eluting stents } \\
\text { Implants }\end{array}$ \\
\hline Inhalation & $\begin{array}{l}\text { Nasal cavity } \\
\text { Lung }\end{array}$ & $\begin{array}{l}\text { Aerosols (solutions and suspensions) } \\
\text { Powders } \\
\text { Liquids }\end{array}$ \\
\hline
\end{tabular}

For disintegration testing, the FDA Guidance recommends the use of the USP (ICH harmonized) disintegration apparatus. This apparatus requires a significantly greater volume of media than would be found in the buccal cavity and has a subjective endpoint especially for products that form pulpy masses or create cloudy solutions. The use of disks for automatic detection can eliminate some of this subjectivity. More recent objective disintegration methods are also being explored which use lower volumes of liquid. Multiple laboratories have employed a texture analyzer which allows a constant force to be applied to a tablet using a solid probe. The disintegration time is determined from a plot of distance traveled by the probe as a function of time (8-12).

Orally administered, rapidly dissolving films are thin, flexible sheets of polymeric material that contain rapidly dissolving API. These are treated as ODTs, i.e., a dissolution test with disintegration is recommended. Basket apparatus can be used with higher sampling frequency at earlier time points for dissolution determination.

\section{Chewable Tablets}

In principle, the test procedure employed for chewable tablets should be the same as that for regular tablets. This concept is based on the possibility that a patient might swallow the dosage form without proper chewing, in which case, the drug will still need to be released to ensure the desired pharmacological action (13). Where applicable, test conditions would preferably be the same as used for conventional tablets of the same active pharmaceutical ingredient, but because of the nondisintegrating nature of the dosage form, there may be a necessity to alter test conditions (e.g., increase the agitation rate) and specifications (e.g., increase the test duration). A recent work has suggested the use of the $\mathrm{Ph}$. Eur. apparatus for medicated chewing gum. Recommendations for methods for testing medicated chewing gums are covered in the section "Dosage Forms Requiring More Work" (3).

\section{Liquid-Filled Capsules}

Liquid-filled capsules can consist of either hydrophilic or lipophilic formulations. In the case of lipophilic formulations, they may or may not include a surfactant for facilitating emulsion formation. The USP recommends a dissolution test procedure using the rotating paddle method with a minimum amount of surfactant, if needed (e.g., dissolution of valproic acid capsules, methoxsalen capsules). If the liquid-filled 
capsule contains a water soluble active substance, then the addition of a surfactant is generally not needed; however, this is a function of solubility of the active pharmaceutical ingredient as well as the formulation itself. The rotating paddle can have disadvantages for some liquid-filled capsule formulations, as it might be difficult to keep the formulation immersed. Also, emulsified formulations might separate at the liquid-vessel-air interface, and/or formulations could adhere to the paddle or beaker walls.

Increasingly, liquid-filled gelatin capsule performance is measured using a simple rupture test. In case the API is dissolved or suspended in a lipid matrix prior or concomitant to drug release testing, predigestion may be needed. This is done with enzymes such as pancreatin (14). The capsules are either tested using the rotating paddle method or in a few cases a disintegration apparatus. The use of the disintegration apparatus together with an enzymatic digestion is described in USP (15). The time necessary for the capsule to rupture is measured. This procedure requires the analyst to closely observe the test until all of the capsules have ruptured.

Other apparatus have also been successfully used, such as the modified dual chamber flow-through cell, as recommended for lipophilic suppositories, the rotating basket (which keeps the formulation immersed, but might result in blocked meshes), or the reciprocating cylinder (which offers good mechanical agitation but a limited media volume).

Especially during the development phase, a range of test media should be used to characterize and understand the formulation characteristics. In the case of lipid-filled capsules, enzymes in addition to surfactants may be necessary to simulate digestion if this is a rate-limiting step for dissolution and absorption in vivo. The advantage of using lipases is that it more closely reflects physiological conditions. The disadvantages are that it can be expensive and labor intensive when used as a routine test, and typically leads to higher variability.

No one single test method is suitable for all liquid-filled capsules. However, the set of available methods described above should enable the selection of an appropriate test in most cases.

\section{Liquid Oral Dosage Forms}

\section{Suspensions}

Pharmaceutical suspensions are liquid preparations consisting of solid particles dispersed throughout a liquid phase in which the particles are not soluble (16). The external phase is an aqueous, organic, or oily liquid phase in which the insoluble internal phase is uniformly dispersed.

\section{Rationale for Drug Release Testing of Suspensions}

Several individual product-specific monographs for suspensions have been included in the USP with some monographs requiring drug release testing (16). From a biopharmaceutical perspective, drug release may be the rate-limiting step for the absorption of oral suspensions with a chance to in vitro-in vivo correlations (IVIVC) (17). Also, drug release testing is required for market release, evaluation of the impact of manufacturing processes on product performance, or substantiation of label claims (18).

\section{Drug Release Methodology}

Suspensions below are grouped according to their routes of administration since it is difficult to identify a single drug release method that would work for all of the suspension types.

\section{Oral Suspensions}

A number of methods have been utilized for the drug release testing from suspensions, but the rotating paddle method using an aqueous medium is recommended for the drug release testing of oral suspensions. The stirring rate may be adjusted to the viscosity of the suspension under test $(1,17-21)$. Higher rates were found to be necessary in case of viscous preparations to prevent sedimentation and accumulation at the bottom of the vessel and to facilitate the discriminative testing of different batches or formulations. Studies have indicated the suitability of the drug release methodology between 50 and $100 \mathrm{rpm}$ (21-23).

Special attention should be paid to the sample preparation and introduction procedure to ensure accurate and repeatable results. Resuspension should be standardized with regard to acceleration, amplitude, frequency, and time course of shaking to provide homogeneity of the samples prior to the transfer of aliquots into the vessel.

A sample introduction technique may vary accompanied with weighing depending on the viscosity, dispersant medium, and the suspending agent used in the formulation (19).

The aliquot used for drug release testing may differ from the therapeutic dose, or the surfactants may be added if required by the solubility of the drug substance in the drug release medium (19).

\section{Topical/Dermal Dosage Forms}

\section{Transdermal Patches}

Although several apparatus and procedures have been utilized to study in vitro release characteristics of transdermal patches, it is desirable to avoid unnecessary proliferation of dissolution/drug release test equipment. Current compendial apparatus include the paddle over disk/disk assembly method (Ph. Eur. 2.9.4.1/USP apparatus 5), the rotating cylinder ( $\mathrm{Ph}$. Eur. 2.9.4.3/USP apparatus 6), the reciprocating disk, and a paddle over extraction cell method (Ph. Eur. 2.9.4.2).

The paddle over disk procedure with a watch glasspatch-screen sandwich assembly could be a suitable method as it has been shown experimentally that this procedure results in almost the same release profile as other, more complicated apparatus for all US marketed transdermal patches (24). The configuration of this assembly ensures that the patch is prevented from floating during the entire testing period. Alternatively, the patch can be fixed to the supporting disk (e.g., by double-sided adhesive), superseding the use of a screen for fixation. Special attention needs to be given to the proper positioning of the patch so that the drug-loaded surface is exposed to the medium.

The $\mathrm{pH}$ of the medium ideally should be adjusted to pH 5-6, reflecting physiological skin conditions. For the same reason, the test temperature is typically set at $32^{\circ} \mathrm{C}$ (even 
though the temperature may be higher when the skin is covered). One hundred revolutions per minute is considered a typical agitation rate by $\mathrm{Ph}$. Eur.

The experimental setup (dissolution medium, agitation speed, etc.) and testing time should take into account the amount of drug administered to the body during the application time of the patch. In cases where drug release cannot be achieved in an appropriate time by using standard aqueous dissolution media, aqueous-organic solvent mixtures can also be used.

\section{Semisolid Topical Dosage Forms}

Semisolid topical dosage forms include creams, ointments, and gels. In vitro drug release from semisolid topical dosage forms has been extensively investigated using the vertical diffusion cell system (Franz cell diffusion system) (25) with a synthetic membrane and to some extent using the enhancer cell (26). Comparative studies indicate that the two types of apparatus generate similar data with some drug products. At present, only limited data are available with the enhancer cell system, and it lacks collaborative or validation data.

Ideally, a sample weight/volume should reflect a typical dose of the product. Depending on the solubility of the drug substance, the receptor medium may need to contain alcohol and/or a surfactant. However, it is preferable to use a partial dose rather than adding a surfactant or alcohol to the receptor medium in order to obtain sink conditions.

De-aeration is critical to avoid bubble formation at the interface with the membrane. A synthetic membrane is often used to serve as an inert support membrane. Depending on the characteristics of the drug product, it may also be possible to conduct the in vitro test without a synthetic support membrane (27). For some ointments, the Franz cell has been used with and without membranes, resulting in no difference in the release rate results. The drug release characteristics usually follow the Higuchi model (28). As with transdermal products, the test temperature is typically set at $32^{\circ} \mathrm{C}$ to reflect the usual skin temperature. Deviations might be justified in the case of products for specific sites of action, e. g., vaginal creams may be tested at $37^{\circ} \mathrm{C}$.

No compendial apparatus, procedures, or requirements for in vitro release testing of semisolid topical dosage forms have been described in relevant pharmacopeias to date. However, FDA's Guidance for Industry on Scale Up and Post-Approval Changes for Semisolid (SUPAC-SS) dosage forms describes the release rate studies using the vertical diffusion cell (Franz cell) procedure and requires in vitro release rate comparison between prechange and postchange products for approval of SUPAC-related changes (29). An in vitro drug release test using the vertical diffusion cell system for semisolid dosage forms has been suggested in USP Pharmacopeial Forum which is in line with FDA's SUPAC-SS (30).

\section{Mucosal}

\section{Chewing Gum}

In the case of chewing gums, the intensity and frequency, with respect to the duration of the experiment, of shearing forces/activities (i.e., "chewing" action) can have a significant influence on drug release rate. The European Pharmacopoeia provides a description of a stainless steel three-piston apparatus, which is required for testing of "medicated chewing gums" (31). The test is typically operated at $37^{\circ} \mathrm{C}$ and at 60 cycles per minute. Test media with a pH 6 are commonly used, since this $\mathrm{pH}$ corresponds to reported (32) saliva $\mathrm{pH}$ values of 6.4 (adults) or 7.3 (children). A second apparatus based on a double piston and a double-walled dissolution vessel has also been proposed for inclusion in the $\mathrm{Ph}$. Eur. as an alternate apparatus, now that it is no longer covered by a patent. Further work is underway to refine the detail on both sets of apparatus in order to incorporate both methods in the chapter.

Experience is growing in the usage of these two sets of apparatus, although the results from the two sets of apparatus are not interchangeable for a given product. The standardization of jaws in order to mimic a chewing action in vivo remains one of the greatest challenges. In particular during development, it is recommended to keep the "chewing residue" for later analysis.

\section{Suppositories}

Drug release mechanisms of suppositories primarily follow either erosional or melting processes depending on whether the matrix is soluble or dispersible in aqueous physiological media or if it melts at body temperature (33). The Ph. Eur. seventh edition requires drug release testing of suppositories for a modified release or for prolonged local action only (33). The partition of compound from the water immiscible fatty base to body fluids may have an influence on the bioperformance (34). Other factors influencing drug release from suppositories are listed in Table II.

A paddle method or continuous flow method are favored for the hydrophilic suppositories with productspecific adjustment of parameters such as paddle rotating speed or flow rate of the medium. Sink conditions should be taken into consideration in designing such a drug release testing method (35). A rotation speed of $50 \mathrm{rpm}$ in the paddle method and a flow rate of $16 \mathrm{~mL} / \mathrm{min}$ in the continuous flow method using a phosphate buffer $\mathrm{pH} 7.4$ at $37^{\circ} \mathrm{C}$ can be used as a starting point in method development for such suppositories.

Lipophilic suppositories may undergo several phases before the release of the API such as softening, deformation melting, or disintegration accompanied by spreading $(36,37)$. The initial phase is the greatest source of variability for in

Table II. Factors Influencing the Drug Release from Suppository Formulations (36)

\begin{tabular}{lll}
\hline Rectal environment & \multicolumn{1}{c}{ Drug substance } & \multicolumn{1}{c}{ Matrix } \\
\hline Fluid volume & Solubility & Composition \\
Composition & Surface properties & Melting behavior \\
$\mathrm{pH}$ & Particle size & Surface tension \\
Buffer capacity & Drug concentration & Rheological behavior \\
Surface tension & $\mathrm{pKa}$ & \\
Viscosity & & \\
Luminal pressure & & \\
\hline
\end{tabular}


vitro testing of drug release due to the variability in the surface area exposed to the medium $(37,38)$. Secondly, variability is introduced via the partitioning of the drug in solution and molten and dispersed matrix $(34,38,39)$. For drug release testing of lipophilic suppositories, it is recommended in $\mathrm{Ph}$. Eur. to employ continuous flow apparatus (34). Nevertheless, if reproducibility of test results is given, a basket or paddle apparatus can also be used.

Intrauterine devices are addressed under implants. Nasal aerosol products are designed to deliver drug through nasal mucosa, and are discussed under Aerosols/ Inhalation products.

\section{Parenteral Dosage Forms}

\section{Intravenous, Subcutaneous, and Intramuscular}

Parenterals formulated as microparticulate systems are intended for the controlled or modified release of the drug substance in which drug release process continues over periods of weeks, months, or even years. The in vivo conditions at the site of injection such as body temperature, metabolism, tissue $\mathrm{pH}$, buffer capacity, level of exercise as well as the volume and osmolarity of the product are to be considered with regard to performance indicating drug release testing methods $(18,40)$. Since real-time methodology mimicking in vivo conditions take months for drug release to occur, special attention should be paid to evaporation and contamination of the media.

In vitro drug release testing methodology for dispersed systems can be grouped into three major categories: sample and separate technique $(1,22)$, membrane diffusion technique (dialysis sac), and continuous flow-through technique $(41,42)$.

The dialysis sac method or the rotating dialysis cell model is mostly preferred because the media and the particles are already physically separated by a membrane, and there is no need for extra separation before the sample measurement as well as for the retention of specimens in the system $(41,43)$. This in vitro model may constitute a valuable tool for describing the effect of drug and formulation characteristics on the drug release rate from oil-based suspensions and to describe the in vitro release and transport processes in a quantitative manner. The suitability of this method for the long-term and accelerated short-term release testing has been demonstrated for Leuprolide-containing poly(lactide-co-glycolide) (PLGA) microspheres (42). In some situations, barrier techniques like the rotating dialysis bag method can lead to masked results due to the limited membrane surface area available for transport from the donor to the receiver compartment compared with the surface area available for transport from the dispersed phase droplets to the continuous phase $(41,44)$.

Continuous flow methodology with proper adjustment of the parameters such as media, flow rate, and sample cell type is mostly suggested for the drug release testing of parenterals $(18,41)$. The applicability of the flow-through cell has been successfully evaluated using the dexamethasone-containing PLGA microspheres $(22,45)$. With the continuous flow-through method, aggregation of the hydrophobic microspheres, media evaporation, loss of microspheres during sampling, and media replacement and operator variability could be minimized (22).
Accelerated tests should have relevance to the real-time tests and should not alter the mechanisms of the drug release but only speed up the process. Thus, the quality control of extended-release preparations can be conducted with respect to biorelevance $(18,40,43)$. Especially, such a release method should be able to identify burst release from the formulation and also supply information about the duration of this phase for controlling the efficacy and safety of the product. In general, it was considered that in vitro release of over $80 \%$ is desirable. Such a method can be developed through the modifications of $\mathrm{pH}$, temperature, or physical agitation (43).

\section{Inhalation Products/Aerosols}

At present, there is no in vitro drug release test for aerosol products. However, this section is included for completeness of performance test for all dosage forms based on taxonomy.

Drug products administered as aerosol falls into two general categories: those delivered by oral inhalation (oral inhalation aerosols) and those delivered through the nasal cavity (nasal aerosols). Oral inhalation aerosols are intended to produce fine particles or droplets for inhalation through the mouth and deposition in the pulmonary tree. The design of the delivery system releases one dose with each actuation. These products are commonly known as metered dose inhalers or dry product inhalers. Nasal inhalation aerosols produce fine particles or droplets for inhalation through the nasal vestibule and deposition in the nasal cavity. Two most important criteria to assess performance of these products are (1) aerodynamic particle size distribution (APSD) and (2) uniformity of dose delivered. The APSD are traditionally assessed by multistage cascade impactors. This gold standard method provides direct measures of particle size in terms of aerodynamic diameter and enables the mass of active pharmaceutical ingredient (API) to be quantitated traceably.

\section{DOSAGE FORMS REQUIRING MORE WORK BEFORE A METHOD CAN BE RECOMMENDED}

\section{Oral}

\section{Powders, Granules, Solid Solutions, and Solid Dispersions}

It is important to note that the dissolution behavior of these dosage forms may be greatly influenced by their wettability, surface area, and particle size distribution. Thus, the in vitro dissolution test results constitute one of a group of physicochemical parameters needed to characterize the product. For powders, especially when exhibiting poor wettability, it may be necessary to add a surfactant to the dissolution medium to obtain reproducible dissolution results. Care should be taken to use a level of surfactant that does not increase the solubility of the drug to the extent where the test is no longer discriminatory. In certain cases, a physical mixture of the powder with glass beads and/or substances, which encourage wetting, may be used. A paddle apparatus is proven to be suitable when rotating with a higher rotating speed and immersing the sampling unit permanently. The flow-through cell offers specific 
sample cells for studying dissolution from powder and granular dosage forms.

Solid solutions and dispersions may be presented in oral dosage forms such as capsules and tablets. If this is the case, their in vitro release characteristics can be determined using the same methods typically used to characterize the release from solid oral dosage forms. Solid solutions and dispersions often lead to a supersaturation of the medium. Therefore, for these specific types of formulations, dissolution tests under non-sink conditions can be a predictive tool during formulation development as well as for batchto-batch quality control. Especially during product development, running the in vitro release test somewhat longer, e.g., for up to $4 \mathrm{~h}$, should be considered to assess the potential for precipitation (1).

\section{Mucosal}

\section{Thin Dissolvable Films (Wafers)}

Wafers are dosage forms designed to attach to mucosal surfaces and release the drug to or through the mucosa either for local or systemic action. They can be used for application, e.g., in the oral cavity or to the vagina.

Wafers intended for administration to the oral cavity may partially or completely be swallowed after disintegration, and thus can result in buccal and/or GI absorption. So both dissolution and disintegration testing may be relevant.

Depending on the delivery strategy, quality control tests including disintegration and/or dissolution testing are required to be developed. Traditional methods and apparatus for measurement of drug release profiles are designed to simulate the gastrointestinal tract. With respect to the conditions of the oral or vaginal mucosa, low-volume test methods may be discriminative and suitable to reflect the in vivo conditions. For these products, basket apparatus can be used with frequent and higher sampling at early time points.

\section{Opthalmic Dosage Forms}

Difficulties encountered when delivering medication to the eye are numerous. The cornea presents a significant barrier to not only noxious chemicals, but also beneficial drugs. Achieving the therapeutic levels of a drug in the interior of the eye is particularly challenging. Drugs containing inserts directly bypass the corneal barrier but require surgical implantation. Dosage forms containing mucoadhesives act to extend formulation residence time in the eye. Similar to some oral dosage forms, cyclodextrins have been used to enhance drug solubility; however, some cyclodextrins are toxic to the cornea.

Once a delivery strategy has been selected, various quality control tests, sometimes including dissolution testing, are required to be developed. Traditional methods and apparatus for the measurement of drug release profiles are designed to simulate the gastrointestinal tract and are inappropriate for ophthalmic dosage forms. Even low-volume variants of the compendial apparatus do not approximate the conditions in the eye. The development of unique tests, equipment, and specifications is frequently required for ophthalmic dosage forms. Convection diffusion is especially relevant to flow-through cell apparatus techniques. The utilization of appropriately modified instrumentation, techniques, and methods has resulted in vastly improved precision, formulation relevant sensitivity, and analytical sensitivity. This has also yielded much greater mathematical modeling and predicting capabilities.

\section{Parenterals}

While the need to demonstrate in vitro performance is recognized by regulatory authorities (46-48), currently there is no consensus specifying release testing methodologies for parenteral products.

\section{Drug-Eluting Stents}

Coronary stents are implantable devices that are placed percutaneously in one or more coronary arteries to maintain patency. Drug-eluting stents (DES) incorporate a pharmacologically active agent (drug) that is delivered at the site of stent deployment and is intended to reduce the incidence of restenosis due to neointimal hyperplasia associated with bare metal stenting. In many cases, the drug is incorporated into and released from a polymeric coating, which modulates its delivery at the intended site of action and for the intended duration. Thus, DES are combination products, consisting of a device and a drug product. Ideally the in vitro drug elution test method should mimic the in vivo drug elution profile of the drug from the DES, although time scaling may be employed to reduce the time of the test. However, faster in vitro release methods are also being developed for Quality Control testing. The in-vivo data are usually obtained from an animal model by determining the drug concentration levels in blood and in the tissues surrounding the DES, and/or by measuring the amount of drug remaining in the polymeric matrix of the DES after stent removal. The in vitro drug release method should capture the beginning, middle, and end of the drug elution profile and should be carried out until a plateau of drug elution is released or at least $85 \%$ of the drug is eluted. The amount of the released drug in vitro is reported as the percentage of the label claim. Suitable instruments for the in vitro drug release of DES are paddle apparatus, flow-through cell, or reciprocating holder. Modification to the compendial testing equipment may be necessary. The development of acceptable IVIVC models is feasible for DES.

Small-volume dissolution apparatus, volume 1-4 mL, has been used to measure drug release from medical devices such as drug-eluting stents, including instruments featuring magnetically driven reciprocation mechanism and heater jackets.

\section{Implants}

Implants are usually solid polymeric devices with a drug load and a release mechanism that ensures the amount of drug being delivered per time unit throughout their residence time in the body. They may be biodegradable and may require medical assistance for insertion and removal if necessary. They may either act locally or systemically. The residence time varies from days to years. The difference from 
the medical devices is that the drug release, rather than the physical effect, is their primary purpose.

Similar to oral dosage form dissolution, an in vitro release test for implants must be established as early as possible in the development process. A detailed description of the development of the in vitro release methodology is required and should include a representative data set as well as an internal validation of the drug release method predictability. Where possible, method development should occur based on real-time data. With the given variability of biological models, a combination of blood level data with residual content of implants after removal may be necessary. In cases where extended time requirements are necessary for real time experiments, mathematical models for extrapolation are allowed. While accelerated methods may be acceptable for quality control purposes, the validation information should demonstrate that the selected test is discriminating and able to detect meaningful manufacturing changes. A relation between real time in vitro release and accelerated in vitro release data is encouraged and should apply when setting acceptance criteria for the quality control method (49).

The in vitro drug release test should predict human in vivo performance (50). If so, it may be used as a surrogate of in vivo bioequivalence for certain pre- or post-approval CMC-related changes.

\section{Microparticulate, Nanoparticulate, and Liposome Formulations}

The paddle apparatus, flow-through cell, and modified flow-through cell have been used successfully for these formulations. The compendial flow-through apparatus is modified with regard to the inner diameter to suit the special properties for testing parenterals, i.e., a low volume of fluid is used in the acceptor compartment. The flow rate of the medium has to be set very slow. The use of HPLC pumps may be considered to provide the necessary accuracy and precision at very low flow rates. In this case, the flow-through system may need to be redesigned with small internal diameter tubing. An intermittent flow might also be an option. The incorporation of glass beads in the flow-through cell may help minimize agglomeration during testing, and cell size may help in the discrimination of a critical formulation or process variables. Static or rotating bottles have also been used for in vitro release testing. Possible alternatives to the standard paddle in vitro release test include utilization of the dialysis sac versus sample and separation.

For microparticle/nanoparticle formulations, blockage and reproducibility for membrane systems are a concern. As tests are often run over a long time period (e.g., several weeks to months), measures have to be taken to compensate against evaporation. Suitable preservatives may be added to prevent microbial contamination. Standard preservatives, including cetylammonium bromide, benzalkonium chloride, parabens, phenol derivatives, and mercury salts, along with appropriate concentrations to be used, are listed in many pharmaceutical textbooks. The selection has to be based on criteria such as compatibility with the active pharmaceutical as well as other formulation ingredients and the $\mathrm{pH}$ of the test medium. Issues with these compounds include their ionization properties, physicochemical interactions, or analytical interferences.

The composition of the medium should take into consideration the osmolarity, $\mathrm{pH}$, and buffer capacity of the fluids at the site of administration, which are usually assumed to resemble that of plasma (or muscle) but with lower buffer capacity. However, the main challenges with this type of dosage form are to determine the appropriate duration of the test and the times at which samples are to be drawn in order to characterize the release profile adequately. The possibility of running the test under accelerated conditions is attractive and has been successfully applied through elevated test temperatures (even above glass transition temperatures of the polymers involved) and at $\mathrm{pH}$ values offering faster drug release (51).

To evaluate whether accelerated test data are predictive, the Weibull shape factor could be considered (52). The verification of the validity of using accelerated test conditions could also include an Arrhenius plot after obtaining release rate constants from linearized release profiles (53).

For real-time (long duration) and accelerated tests, employing potentially adverse temperatures or $\mathrm{pH}$ values, the stability of the active ingredient has to be taken into account either analytically or through appropriate algorithms when calculating release data.

Both paddle apparatus and flow-through cell can be used for determining drug release from microsphere formulations. However, flow-through cell is preferable and less problematic in handling microspheres and liposome samples. The flowthrough cell with a slight modification of the holding cell has been successfully used to study drug release profile from liposomes (54).

\section{Inhalation}

At present, there is no official in vitro drug release method for aerosol products. However, this problem has been studied for at least 30 years (55). There is potential value for in vitro setups that could provide information on drug release from the inhaled particles/droplets onto the lung lining mucosa. Davies et al. designed a flow-through cell apparatus to measure dissolution rates from glass fiber filters that had been placed within an Andersen Cascade Impactor (ACI) (56) and, more recently, Son et al. (57) used a Next Generation Pharmaceutical Impactor (NGI) modified by placing wax paper in the collection cups to collect the sized fractions. The wax paper pieces were then placed into plastic histology cassettes which were dropped into vessels of a paddle apparatus. More recently, this same research group modified an NGI with removable impaction cups that could then be dropped into the paddle apparatus (58). In the past few months, two separate research groups have demonstrated the use of diffusion apparatus to assess dissolution rates for inhalables. Arora et al. placed small PVDF membrane filters on upturned ACI impaction plates then transferred the membranes to a Transwell-type diffusion apparatus (59), whereas Forbes et al. (60) used nitrocellulose filter membranes with a Franz-type apparatus. However, the latter group did not fractionate the aerosol prior to dissolution measurement. The use of a diffusion apparatus probably better simulates what occurs within the thin layer of fluid 
within the lungs than does a bulk type apparatus such as the paddle apparatus. As a result, the future of dissolution for testing inhalation devices is under evaluation.

\section{FORMULATION CHARACTERIZATION}

In order to characterize the release from the dosage form adequately, it is recognized that a drug release profile should be generated, in which release (dissolution) values are determined as a function of time. This multipoint characterization has been in place for modified release oral dosage forms for some time and is also recommended for slower dissolving immediate release products. Because many of the dosage forms discussed here are complex in terms of composition and release mechanism, a multipoint drug release test should be required to characterize release from the drug product in general and to test for possible alterations in the release profile during storage. Multipoint tests may also be needed for batch release testing in order to confirm acceptable batch-to-batch consistency. Typical cases where multipoint tests are likely to be needed include transdermal patches, semisolid preparations, chewing gums, implants, microparticulate formulations, solid solutions, solid dispersions, and liposomes. However, in other cases like powders, granules, suspensions, orally disintegrating tablets (unless multipoint testing is used for evaluation of taste masking), chewable tablets, and rapidly releasing suppositories, a single point specification may be sufficient for batch-to-batch quality control. In these cases, the timepoint must be properly derived from profiles generated during the development phase of the product.

\section{EXPERIMENTAL TEST CONDITIONS}

The experimental test conditions should be discriminating enough to detect manufacturing variables that may affect biopharmaceutical product performance. Test conditions that may not be able to discriminate adequately among products/ batches with different in vivo release profiles include those with very high agitation/flow rates, the use of strongly alkaline solutions to dissolve poorly soluble acids, and the use of very high surfactant concentrations to create sink conditions, to name but a few.

As for solid oral dosage forms, the development of in vitro dissolution/release tests and specifications for novel/ special dosage forms should take into account relevant bioavailability or clinical data. However, expectations with respect to the quality and/or level of in vitro/in vivo correlation should not be set as high as for solid oral dosage forms because of the higher level of complexity and data variability for novel/special dosage forms.

As the release mechanism and site of application varies dramatically among the novel/special dosage forms, the experimental test conditions should be tailored according to the conditions at the site of administration (e.g., temperature of the test) and the release mechanism (e.g., chewing gums will require different agitation rates than suspensions). The complexity of the release mechanism of some novel/special dosage forms and the lack of knowledge about the conditions under which release occurs in vivo make it difficult to design physiologically based tests in all cases, but it should be possible to conceive a test that can detect the influence of critical manufacturing variables, differentiate between the different degrees of product performance, and to some extent characterize the biopharmaceutical quality of the dosage form. Within a given category, it may be necessary to have product-type specific dissolution tests (e.g., separate tests for lipophilic and hydrophilic suppositories), and in some cases for products containing the same drug and administered in the same type of novel/special dosage form, but with a different release mechanism (analogous to the range of tests available in the USP for theophylline-extended release dosage forms).

Test procedures for dissolution testing of solid oral dosage forms, i.e., immediate release and modified release dosage forms, have been significantly refined and standardized over the past quarter century.

Because of the slow release characteristics of several complex novel dosage forms (e.g., implants, microspheres, liposomes), it may be necessary to study the release profile at elevated temperatures (making sure that the higher temperature does not destroy the characteristics of the dosage form) for using it as a quality control test.

While compendial options for additional types of dissolution have improved, for novel/special dosage forms more than for solid oral dosage forms, it is difficult to find the appropriate balance between the general recommendation to avoid "unnecessary" proliferation of dissolution apparatus and acknowledging the formulation-specific characteristics and requirements of a new product under development. "Unnecessary" refers to a proliferation of apparatus for a newly developed dissolution test when a comparison of data indicates similarities (or equivalence) with standard compendial equipment. In such situations, clearly the compendial apparatus should be used.

\section{BENEFITS AND APPLICATIONS FOR DISSOLUTION/ DRUG RELEASE}

A specific value of in vitro dissolution/drug release testing is recognized in its application as a batch-to-batch quality control test and its value in evaluation and approval of Scale-Up and Post-Approval Changes (SUPAC). The SUPAC document for semisolid dosage forms (SUPAC-SS) defines the levels of changes with respect to the component and composition, site of manufacturing, scale of manufacturing, and process and equipment changes (29). In vitro drug release is used to assure product sameness for semisolid dosage forms under SUPAC-related changes. The same principles can easily be extended to other dosage forms where the product sameness can be assured by profile comparison between prechange and postchange products using an appropriate in vitro test and profile comparison, e.g., for transdermal patches (47). In addition to this, the dissolution/drug release test can also be used for providing biowaivers for lower strengths of a product from a given manufacturer, once the higher strength is approved based on appropriate bioavailability/bioequivalence test procedure.

Even though less experience is available for novel/special dosage forms compared to conventional dosage forms, in vitro/in vivo correlations have been established and thus are possible. In such cases, it is legitimate and should find support 
from a regulatory perspective to use in vitro dissolution as a surrogate for the in vivo performance of a drug product, as long as the rate-limiting step is the release of the drug from the formulation. Because of the typically higher variability of in vivo and in vitro data in the case of many novel/special dosage forms, expectations towards the quality and level of in vitro/in vivo correlations might have to be adjusted in comparison to "conventional" dosage forms.

In general, an in vitro dissolution/release test is expected for each novel/special dosage form regardless of whether the intended effect is systemic or nonsystemic (e.g., topical semisolid dosage forms), for formulation development, for investigations to support post-approval changes and for batch-to-batch quality control. However, because of the specific formulation design and potential (physicochemical) interactions between the dosage form and the physiological environment at the site of administration, and also because of the necessary design of in vitro dissolution equipment for novel/special dosage forms, dissolution/release data in vitro might be strongly influenced by test or equipment parameters and therefore potentially less predictable for in vivo release than typically experienced for "conventional" dosage forms. Therefore, a scientifically sound assessment of the relevance and validity of an in vitro dissolution test should determine the final decision about the application of the test and setting of specifications for batch-to-batch quality control.

The in vitro drug release test for some novel/special dosage forms such as semisolid dosage forms and transdermal drug delivery systems has proven to be equally valuable as the dissolution test for solid oral dosage forms. The in vitro drug release test also shows promise for other dosage forms, such as chewable tablets, suspensions, and suppositories. For yet other dosage forms, such as chewing gums, powders, and parenterals, further method development and refinement will be needed to make the drug release test a generally applicable, robust, and valuable tool. A summary table providing the typical apparatus used for various dosage forms is provided (Table III).

\section{SETTING SPECIFICATIONS: ACCEPTANCE CRITERIA/LIMITS}

The in vitro dissolution/drug release specifications should be primarily based on manufacturing experience, formulation screening experience, and pivotal clinical trial batches or other biobatches. Compared to testing of solid oral dosage forms in basket and paddle dissolution equipment, far less experience is available for many of the novel/special dosage forms with respect to variability of data and, where the newer types of apparatus are used, qualification of the equipment. In general, criteria and specification limits (ranges) may be set similarly to the procedure for solid oral dosage forms. However, further experience must be gained to better understand the desired level of standardization, and it can be expected that, in some instances, the appropriate ranges and criteria for acceptance of release data of novel/special dosage forms will be very different from those for solid oral dosage forms. Acceptance criteria need to be set on a product specific basis, based on sufficient data to ensure the consistent quality of manufactured batches. Where sufficient experience has been gained advice has been included in the specific sections above to assist in the determination of suitable acceptance criteria for the different product categories

In general, in vitro dissolution/release specifications apply throughout the shelf life of a drug product ("end-of-shelf-life specification"). Nevertheless, acknowledging the nature and design of some novel/special dosage forms, small changes of dissolution/release properties within the shelf life period have to be taken into consideration. Thus, pharmaceutical manufacturers may be well advised to apply separate internal specifications at the time of batch release, if appropriate, which are different, i.e., stricter than formal specifications.

\section{CONCLUSIONS}

An appropriate product quality and product performance (drug release) test is required to characterize the drug product and assure batch-to-batch reproducibility for consistent pharmacological/biological activity.

For oral products, the dissolution test is recognized as a valuable in vitro tool as a measure of performance test. Similarly, for topical and dermal drug products sufficient advances have been made to propose in vitro release test. A paddle over disk is suggested for drug release from transdermal patches or delivery through skin. For semisolid preparations, drug release using vertical diffusion cell assembly is recommended. For other dosage forms, parenterals, and mucosals, a significant progress has been

Table III. Suggested Apparatus for Drug Release Testing of Various Dosage Forms

\begin{tabular}{ll}
\hline \multicolumn{1}{c}{ Dosage form example } & Release method \\
\hline Oral solid dosage forms (conventional) & $\begin{array}{l}\text { Basket apparatus, paddle apparatus, reciprocating cylinder or flow-through cell } \\
\text { Paddle apparatus }\end{array}$ \\
Oral suspensions & $\begin{array}{l}\text { Paddle apparatus and disintegration method } \\
\text { Oral disintegrating tablets }\end{array}$ \\
Chewable tablets & Basket apparatus, paddle apparatus or Reciprocating cylinder \\
Powders and granules & Flow-through cell (powder/granule sample cell) \\
Thin dissolvable films & Basket apparatus and disintegration method \\
Chewing gum & Special apparatus (Ph. Eur.) \\
Dermal delivery systems (patches) & Paddle over disk \\
Topical (semisolid dosage forms) & Franz cell diffusion system \\
Suppositories & Paddle apparatus, modified basket apparatus or dual chamber flow-through cell \\
Microparticulate formulations & Modified flow-through cell \\
Implants & Modified flow-through cell \\
Aerosols & Cascade impactor \\
\hline
\end{tabular}


made towards the development of drug release from the formulations. However, more work needs to be performed and validated before a standard method/test can be proposed. For inhalation products, the important tests are measuring aerodynamic particle size distribution and uniformity of dose delivered.

\section{ACKNOWLEDGMENTS}

This revision of this publication represents the scientific opinion of many experts and in particular, is derived from two key workshops (London, 2008 and Los Angeles, 2009) held under the auspices of the International Federation of Pharmaceutical Sciences (FIP) with cosponsorship from the Royal Pharmaceutical Society of Great Britain (RPSGB), American Association of Pharmaceutical Sciences (AAPS), and the US Food and Drug Administration (FDA). The authors would like to acknowledge the following workshop presenters for their technical contributions to the workshops which were fundamental to the revision of this publication.

International Pharmaceutical Federation (FIP) Workshop, Special dosage forms: What's new with in vitro drug release? Cosponsored with the Royal Pharmaceutical Society of Great Britain (RPSGB), London, UK, 20-21 October 2008

-Akinari Abe, Taisho Pharmaceutical Co., Japan: "In Vitro Release of Suppositories"

-Lori Alquier, Cordis Corporation, USA: "Elution Testing of Cardiovascular Stents: An Industry Perspective"

-Lucinda Buhse, CDER/FDA, USA: "Orally Disintegrating Tablets or Orodispersible Tablets"

-Todd Cecil, USP, USA: "Overview of USP Dosage Form Taxonomy"

-Neal Davies, Washington State University, USA: "Is it Time for Dissolution Testing of Inhalation Dosage Forms?"

-Angelica Dorantes, CDER/FDA, USA: "Drug Eluting Stents-Regulatory Perspective"

- Jennifer Dressman, University of Frankfurt, Germany: "Overview of Current FIP Solid Dosage Forms White Paper" -Munir Hussain, Bristol Meyer Squibb, USA: "Release Testing for Special Dosage Forms: Nasal and Intra-Oral Products"

-Susanne Keitel, EDQM, Council of Europe: "Dissolution Testing of Special Dosage Forms-The European Point of View"

-Dr Hiroshi Kikuchi, Eisai Co., Japan: "The Meaning of InVitro Drug Release in the Case of Liposomes Which must be Delivered to Target Organs/Cells"

- Jayachandar Gajendran, PHAST, Germany: "Performance Testing of Medicated Chewing Gums"

- Roy De Maesshlack, Johnson \& Johnson, Belgium: "Intramuscular Nano Suspension Method Development with USP Apparatus IV (Flow Through)"

-Siddhesh Patil, Amylin, USA: "Microspheres"

-Larry Stevens and Paul Missel, Alcon Research, USA: "Dissolution in Ophthalmics: A High-Performance Approach" -Clarence Ueda, University of Nebraska, USA: "Performance Testing of Topical Dosage Forms"
AAPS Workshop: Special dosage forms-what's new with in vitro drug release? Cosponsored with FDA and FIP Los Angeles, CA, USA, 7-8 November 2009

-Kent Alleman, Ph.D., Bausch \& Lomb: "Performance Tests for Ophthalmics: Solution, Suspension, Emulsion"

-Lori Alquier, Ph.D., Cordis Corporation: "Performance Tests for Drug-Eluting Stents: Industry Perspective"

-Cynthia Brown, Eli Lilly and Company: "Summary Report of London Workshop on In Vitro Release of Special Dosage Forms"

-Lucinda Buhse, Ph.D., U.S. Food and Drug Administration: "Orally Disintegrating Tablets-Disintegration vs. Dissolution"

-Diane Burgess, Ph.D., University of Connecticut: “In Vitro Release of Injectables-Academic Perspective"

-Angelica Dorantes, Ph.D., U.S. Food and Drug Administration: "Performance Tests for Drug-eluting Stents: Regulatory Perspectives"

- Horst-Dieter Friedel, Ph.D., Bayer Schering Pharma AG: "Conclusions and Next Steps"

-Anthony Hickey, Ph.D., University of North Carolina: "Performance Tests for Aerosol Products: Oral Inhalation"

- Henk de Jong, Ph.D., European Pharmacopoeia Commission: "International Perspectives on the Drug Release of Special Dosage Forms-The European Pharmacopoeia"

-Patrick J. Marroum, Ph.D., U.S. Food and Drug Administration: "In Vitro Release of Injectables-Regulatory Perspective"

-Gordon McKay, Ph.D., Pharmalytics Inc.: "Chewable Oral Drug Products"

-Jolyon Mitchell, Ph.D., Tredall Medical International: "Performance Tests for Aerosol Products: Nasal"

-J. Michael Morris, Ph.D., Irish Medicine Board: "International Perspectives on the Drug Release of Special Dosage Forms"

-Thomas E. Redelmeier, Ph.D., Northern Lipids: “Targeted Drug Delivery with Liposomes"

-Vinod P. Shah, Ph.D., FIP Scientific Secretary: "Introduction and Product Performance Tests for Special Dosage Forms"

-Larry Stevens, MS, Larry Stevens Consulting : "Performance Tests for Ophthalmics: Inserts"

-Mary Stickelmeyer, Ph.D., Eli Lilly and Company: "In Vitro Release of Injectables-Industry Perspective"

- Roger Williams, M.D., United States Pharmacopeia: "International Perspectives on the Drug Release of Special Dosage Forms"

\section{REFERENCES}

1. Siewert M, Dressman J, Brown C, Shah VP. 2003. FIP/AAPS guidelines for dissolution/in vitro release testing of novel/special dosage forms. Dissolution Technologies. 2003;10(1):6-15.

2. International Pharmaceutical Federation (FIP) Workshop, special dosage forms-what's new with in vitro drug release? Cosponsored with the Royal Pharmaceutical Society of Great Britain (RPSGB), London, UK 20-21 October, 2008.

3. AAPS workshop: special dosage forms - what's new with in vitro drug release? Cosponsored with FDA and FIP Los Angeles, CA, USA, 7-8 November 2009.

4. FIP guidelines for dissolution testing of solid oral products, Die Pharmazeutische Industrie 59: 760-766 (1997) and Dissolution Technologies 4:5-14 November (1997). 
5. FDA guidance for industry: dissolution testing of immediate release solid oral dosage forms, August 1997, extended release solid oral dosage forms: development, evaluation and application of in vitro/in vivo correlations, September 1997; and Dissolution Technologies 4: 15-22 and 23-32, November 1997.

6. Marshall K, Foster TS, Carlin HS, Williams RL. USP Pharmacopeial Forum. 2003;29(5):1742-52.

7. US Food and Drug Administration. FDA guidance for industry on orally disintegrating tablets. Silver Spring: FDA; 2008.

8. Dor PJM, Fix JA. In vitro determination of disintegration time of quick-dissolve tablets using a new method. Pharm Dev Technol. 2000;5(4):575-7.

9. El-Arini SK, Clas S-D. Evaluation of disintegration testing of different fast dissolving tablets using the texture analyzer. Pharm Dev Technol. 2002;7(3):361-71.

10. Bohnackner R, Streil F, Schweizer S, Müller I. Determination of the disintegration time of mouth melt tablets using the texture analyzer method (English translation). Pharm Ind. 2005;67 (3):327-35.

11. Abdelbary G, Eouani C, Prinderre P, Joachim J, Reynier J, Piccerelle P. Determination of the in vitro disintegration profile of rapidly disintegrating tablets and correlation with oral disintegration. Int J Pharm. 2005;292(1-2):29-41.

12. Narazaki R, Harada T, Takami N, Kato Y, Ohwaki T. A new method for disintegration studies of rapid disintegrating tablet. Chem Pharm Bull. 2004;52(6):704-7.

13. FDA guidance for industry: bioavailability and bioequivalence studies for orally administered drug products-general considerations, October 2000.

14. Kaukonen AM, Boyd BJ, Charmann WN, Porter CJH. Pharm Res. 2004;21(2):254-60.

15. Marques MRC et al. Pharmacopeial forum. 2009;35(4):2.

16. USP 32-NF 27. United States Pharmacopeial Convention, ed., Rockville, MD.

17. Azam MG, Haider SS. Evaluation of dissolution behavior of paracetamol suspensions. Pharmaceutical Science. 2008;7 (1):53-8.

18. Burgess DJ, Crommelin DJ, Hussain AS, Chen ML. Assuring quality and performance of sustained and controlled release parenterals: EUFEPS workshop report. AAPS J. 2004;6 (1):100-11.

19. USP 32-NF 27. General Monographs: Phenytoin Oral Suspension, United States Pharmacopeial Convention, ed., Rockville, MD.

20. Junyaprasert VB, Manwiwattanakul G. Release profile comparison and stability of diltiazem-resin microcapsules in sustained release suspensions. Int J Pharm. 2008;352(1-2):81-91.

21. Larsen SW, Frost AB, Østergaard J, Marcher H, Larsen C. On the mechanism of drug release from oil suspensions in vitro using local anesthetics as model drug compounds. Eur J Pharm Sci. 2008;34(1):37-44.

22. Zolnik BS, Raton J-L, Burgess DJ. Application of USP apparatus 4 and in situ fiber optic analysis to microsphere release testing. Dissolution Technologies. 2005;12(2):11-4

23. Wong J, Brugger A, Khare A, Chaubal M, Papadopoulos P, Rabinow B, et al. Suspensions for intravenous (IV) injection: a review of development, preclinical and clinical aspects. Adv Drug Rev. 2008;60(8):939-54.

24. Shah VP, Tymes NW, Skelly JP. In vitro release profile of clonidine transdermal therapeutic systems and scopolamine patches. Pharm Res. 1989;6:346-51.

25. Shah VP, Elkins JS, Williams RL. Evaluation of the test system used for in vitro release of drugs from topical dermatological drug products. Pharm Develop Technology. 1999;4:377-85.

26. Fares HM, Zatz JL. Measurement of drug release from topical gels using two types of apparatus. Pharm Tech. 1995;19(1):52-8.

27. Shah VP, Elkins JS. In vitro release from corticosteroid ointments. J Pharm Sci. 1995;84:1139-40.

28. Higuchi WI. Analysis of data on medicament release from ointments. J Pharm Sci. 1962;51:802-4.

29. FDA Guidance for Industry: SUPAC-SS nonsterile semisolid dosage forms. scale-up and post approval changes: chemistry, manufacturing, and controls; in vitro release testing and in vivo bioequivalence documentation, May 1997.
30. USP. Pharmacopeial Forum. Pharmacopeial Forum. 2009;35 (3):602-11. 615-626.

31. Council of Europe (2002) European pharmacopoeia 4th edn. In: Chewing gum medicated drug release form. Council of Europe, Strasbourg, pp 227-228.

32. Diem K, Leutner C, editors. Documenta Geigy, scientific tables 7th edition. New Jersey: Geigy Pharmaceuticals; 1975. p. 643.

33. Council of Europe. European pharmacopoeia, 7th edition. Strasbourg: Council of Europe; 2011.

34. Loyd VA, editor. Suppositories, first edition. London: Pharmaceutical Press; 2007.

35. Ibrahim SA et al. Formulation, release characteristics and evaluation of ibuprofen suppositories. Int J Pharm. 1990;61:1-7.

36. de Blaey CJ, Fokkens JG. Drug release from suppositories. Pharm Res. 1985;2(2):61-4.

37. Roseman TJ et al. Continuous flow bead-bed dissolution apparatus for suppositories. J Pharm Sci. 1981;70(6):646-51.

38. Janicki $\mathrm{S}$ et al. Evaluation of paracetamol suppositories by a pharmacopoeial dissolution test-comments on methodology. Eur J Pharm Biopharm. 2001;52(2):249-54.

39. Woyczikowski B et al. Feasibility of the Ph. Eur. flow-through cell for dissolution testing of the compounded rectal suppositories containing indomethacin or sodium diclofenac. Acta Pol Pharm. 2003;60(3):169-72.

40. Burgess DJ, Hussain AS, Ingallinera TS, Chen ML. Assuring quality and performance of sustained and controlled release parenterals: AAPS workshop report, co-sponsored by FDA and USP. Pharm Res. 2002;19(11):1761-8.

41. D'Souza SS, DeLuca PP. Methods to assess in vitro drug release from injectable polymeric particulate systems. Pharm Res. 2006;23(3):460-74.

42. D'Souza SS, Faraj JA, DeLuca PP. A model-dependent approach to correlate accelerated with real-time release from biodegradable microspheres. AAPS PharmSciTech. 2005;6(4): E553-64.

43. Martinez M, Rathbone M, Burgess D, Huynh M. In vitro and in vivo considerations associated with parenteral sustained release products: a review based upon information presented and points expressed at the 2007 Controlled Release Society Annual Meeting TM. J Control Release. 2008;129:79-87.

44. Chidamabaram N, Burgess DJ. A novel in vitro release method for submicron-sized dispersed systems. AAPS Pharm Sci. 1999;1 (3):32-40.

45. Patil SD, Papadimitrakopoulos F, Burgess DJ. Dexamethasoneloaded poly(lactic-co-glycolic) acid microspheres/poly(vinyl alcohol) hydrogel composite coatings for inflammation control. Diab Technol Ther. 2004;6(6):887-97.

46. FDA Guidance for Industry: Liposome Drug Products. Chemistry, Manufacturing, and Controls; Human Pharmacokinetics and Bioavailability; and Labeling Documentation. Draft, August 2002.

47. Draft Guidance for Industry: Coronary Drug-Eluting StentsNonclinical and Clinical Studies, March 2008.

48. EMEA/CHMP/EWP/110540/2007 Guidelines on the Clinical and Non Clinical Evaluation during the Consultation Procedure on Medicinal Substances Contained in Drugeluting (Medicinal Substance-Eluting) Coronary Stents, 30 May 2008.

49. Karalis V, Magklara E, Shah VP, Macheras P. From drug delivery systems to drug release, dissolution, IVIVC, BCS, BDDCS, bioequivalence and biowaivers. Pharm Res. 2010;27 (9):2018-29.

50. Schliecker G, Schmidt C, Fuchs S, Ehinger A, Sandow J, Kissel $\mathrm{T}$. In vitro and in vivo correlation of buserelin release from biodegradable implants using statistical moment analysis. J Control Release. 2004;94(1):25-37.

51. Shameem M, Lee H, DeLuca PP: A short term (accelerated release) approach to evaluate peptide release from PLGA depot formulations. AAPS PharmSci. 1999;1(3):1-6.

52. Sathe $\mathrm{P}$, Tsong $\mathrm{Y}$, Shah VP. In vitro dissolution profile comparison: statistics and analysis, model dependent approach. Pharm Res. 1996;13:1799-803.

53. Makino K, Arakaw M, Kondo T. Preparation and in vitro degradation properties of Polylactide microcapsules. Chem Phar Bull. 1985;33(3):1195-201. 
54. Bhardwaj U, Burgess DJ. A novel USP apparatus 4 based release testing methods for dispersed systems. Int $\mathrm{J}$ of Pharmaceutics. 2010;388(1-2):287-94.

55. Kanapilly GM, Raabe OG, Goh CH, Chimenti RA. Measurement of in vitro dissolution of aerosol particles for comparison to in vivo dissolution in the lower respiratory tract after inhalation. Health Phys. 1973;24(5):497-507.

56. Davies NM, Feddah MR. A novel method for assessing dissolution of aerosol inhaler products. Int J Pharm. 2003;255(1-2):175-87.

57. Son Y-J, McConville JT. Dissolution testing for inhalation formulations. Inhalation Magazine. 2008;2(6):8-11.
58. Son Y-J, Horug M, Copley M, McConville J. Optimization of an in vitro dissolution test method for inhalation formulations. Dissolution Technologies, 2010. p 6-13.

59. Arora D, Shah K, Halquist M, Sakagami M. In vitro aqueous fluid-capacity-limited dissolution testing of respirable aerosol drug particles generated from inhaler products. Pharm Res. 2010;27(5):786-95.

60. Forbes B, Colombo P, Brambilla G, Sanders M, Jones SA, Buttini F. Important considerations regarding the bioequivalence of particles emitted from beclomethasone dipropionate solution metered dose inhaler. Orlando: RDD; 2010. 